\title{
Suppression of riboflavin-sensitized singlet oxygen generation by L-ascorbic acid, 3- $O$-ethyl-L-ascorbic acid and Trolox
}

\author{
Ryohei Shimizu, Mikio Yagi*, Azusa Kikuchi* \\ Department of Chemistry, Graduate School of Engineering Science, Yokohama National University, Tokiwadai, \\ Hodogaya-ku, Yokohama 240-8501, Japan.
}

\begin{abstract}
ABSTARCT
Riboflavin (RF), a water-soluble vitamin $\mathrm{B}_{2}$, is an endogenous singlet oxygen photosensitizer in human skin and eye. Time profiles of the near-infrared phosphorescence of singlet oxygen generated by RF have been measured in the absence and presence of L-ascorbic acid (AA, vitamin C), 3-O-ethyl-L-ascorbic acid (3-EtAA) and Trolox (TX, a water-soluble analogue of vitamin E) in phosphate buffer ( $\mathrm{pH}$ 6.8). These substances suppress the RF-photosensitized singlet oxygen generation. For example, the quantum yield of singlet oxygen generation is decreased to a third by adding $0.4 \mathrm{mmol} \mathrm{dm}^{-}$ ${ }^{3}$ AA or TX (the concentration of dissolved oxygen in air-saturated water is $0.27 \mathrm{mmol} \mathrm{dm}^{-3}$ ). AA and TX are more efficient suppressors of RF-photosensitized singlet oxygen generation than 3-EtAA. The bimolecular rate constants for quenching of the excited singlet and triplet states of RF by AA, 3-EtAA and TX have been determined through measurements of fluorescence and transient absorption. These measurements suggest that the observed suppression is due to the quenching of the excited singlet and triplet states of RF by AA, 3-EtAA and TX. The bimolecular rate constants for quenching of singlet oxygen by AA, 3-EtAA and TX were determined to be $1.8 \times 10^{8}, 0.27 \times 10^{8}$, and $4.4 \times 10^{8} \mathrm{~mol}^{-1}$ $\mathrm{dm}^{3} \mathrm{~s}^{-1}$, respectively.
\end{abstract}

Keywords: Singlet oxygen; Riboflavin; L-ascorbic acid; 3-O-ethyl-L-ascorbic acid; Trolox

Corresponding authors.

E-mail addresses: yagi-mikio-sc@ynu.ac.jp (M. Yagi), kikuchi-azusa-rh@ynu.ac.jp (A. Kikuchi)

\section{Introduction}

Riboflavin (RF), a water-soluble vitamin $\mathrm{B}_{2}$, is a very efficient endogenous singlet oxygen photosensitizer with a quantum yield of singlet oxygen generation $\Phi_{\Delta}=0.58$ 0.61 [1]. RF is ubiquitous in the animal and plant kingdoms [2]. RF is widely distributed in human skin, eye, cerebral cortex, pectoral muscle, aortic tissue, erythrocyte and plasma [2-5]. In daily life, the skin and eye are frequently exposed to light that origin from solar radiation and artificial light. Singlet oxygen, ${ }^{1} \mathrm{O}_{2}\left({ }^{1} \Delta_{\mathrm{g}}\right)$, generated by endogenous photosensitizers may cause skin damage including photoaging and skin malignancies [6]. Singlet oxygen also may cause damage to the eye lens, cornea and retina [7]. To prevent the deleterious effects of singlet oxygen on health, physical and chemical quenching of 

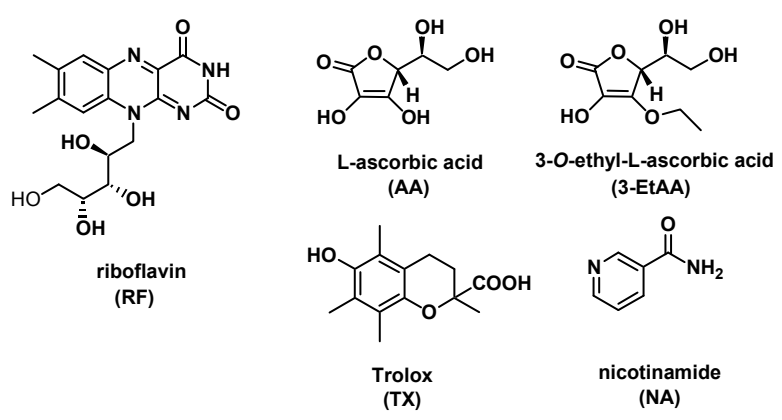

Scheme 1. Molecular structures of RF, AA, 3-EtAA, TX and NA.

singlet oxygen by various substances have been studied [8-11].

L-ascorbic acid (AA, a water-soluble vitamin C, Scheme 1) and Trolox (TX, an analogue of vitamin E, Scheme 1) are well-known water-soluble antioxidants. The bimolecular rate constants for quenching of singlet oxygen by AA and TX have been reported [12-18].

In the present study, we have studied the effects of AA, 3-O-ethyl-L-ascorbic acid (3-EtAA, an analogue of vitamin C, Scheme 1) and TX on the photosensitized singlet oxygen generation by $\mathrm{RF}$ in phosphate buffer $(\mathrm{pH}$ 6.8) at room temperature. 3-EtAA is chemically stable and more easily absorbed through human skin than AA $[19,20]$. 3-EtAA has skin-whitening properties and is used in skinlightening cosmetics [19-22].

Singlet oxygen is the lowest excited state of molecular oxygen. Singlet oxygen shows a characteristic phosphorescence band at $1274 \mathrm{~nm}$ [23]. During the course of the near-infrared (near-IR) phosphorescence studies, the suppression of the RF-photosensitized singlet oxygen generation by AA, 3-EtAA and TX has been observed. The suppression mechanisms are discussed through measurements of fluorescence and transient absorption. The effect of nicotinamide (NA, a water-soluble vitamin $\mathrm{B}_{3}$, Scheme 1) on the RF-photosensitized singlet oxygen generation has been studied for comparison.

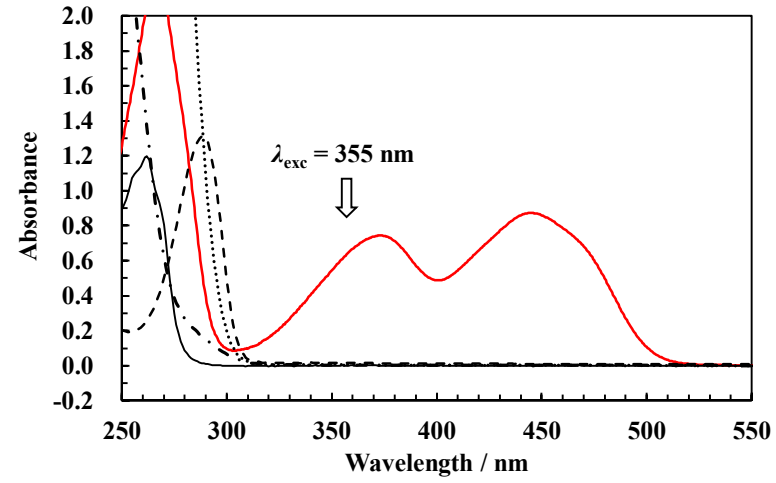

Fig. 1. UV absorption spectra of (a) $0.1 \mathrm{mmol} \mathrm{dm}^{-3} \mathrm{RF}$ (red), (b) 0.4 mmol dm ${ }^{-3}$ AA (dotted line), (c) $0.4 \mathrm{mmol} \mathrm{dm}^{-3} 3$-EtAA (dashdot-dash line), (d) $0.4 \mathrm{mmol} \mathrm{dm}^{-3}$ Trolox (broken line) and (e) 0.4 mmol dm${ }^{-3} \mathrm{NA}$ (solid line) in phosphate buffer (pH 6.8) at $25^{\circ} \mathrm{C}$.

RF (TCI GR Grade), AA (FUJIFILM Wako GR Grade, $>99.6 \%$ ), 3-EtAA (TCI, >98.0\%), TX (TCI, >98.0\%), NA (TCI GR Grade, $>99.0 \%$ ), acetonitrile (FUJIFILM Wako GR Grade) and $0.1 \mathrm{~mol} \mathrm{dm}^{-3}$ phosphate buffer solution (FUJIFILM Wako for biochemistry, $\mathrm{pH}$ 6.8) were used without further purification.

\subsection{Optical measurements}

The details of the UV absorption, transient absorption, fluorescence and near-IR phosphorescence measurements are the same as those described previously [24-28]. For the transient absorption and time-resolved near-IR phosphorescence measurements, sample solutions were excited with a Continuum Surelite Nd:YAG laser (355 nm, repetition rate $10 \mathrm{~Hz}$ ). The energy of the laser pulse was lower than $3 \mathrm{~mJ}$ and each time profile was the result of 512 shots to avoid photodecomposition of sample solutions.

All measurements except the determination of the rate constants of singlet oxygen quenching by 3-EtAA were carried out in phosphate buffer solution $\left(\mathrm{pH} \mathrm{6.8)}\right.$ at $25^{\circ} \mathrm{C}$.

\section{Results and discussion}

\subsection{UV absorption spectra of RF, AA, 3-EtAA, TX and NA}

Fig. 1 shows the UV absorption spectra of RF, AA, 3-EtAA, TX and NA in phosphate buffer at room

\subsection{Chemicals}




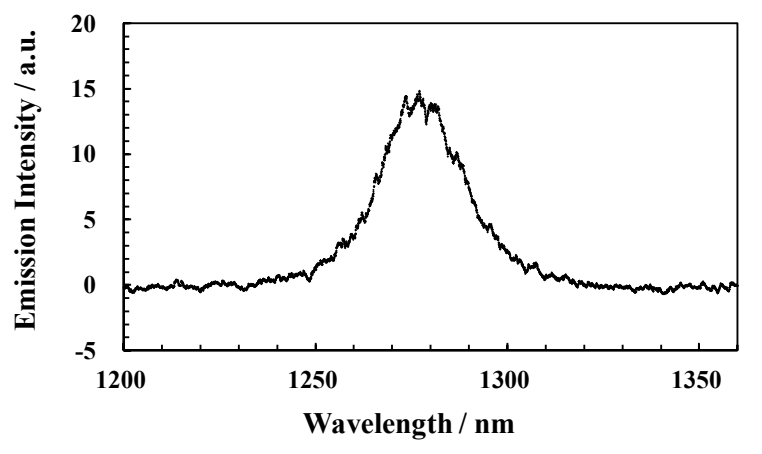

Fig. 2. Time-resolved phosphorescence spectrum of singlet oxygen generated by excitation of RF $\left(0.1 \mathrm{mmol} \mathrm{dm}^{-3}\right)$ in airsaturated phosphate buffer ( $\mathrm{pH} 6.8$ ) at $25^{\circ} \mathrm{C}$. The sampling times were set at $1.0-5.0 \mu$ s after the $355 \mathrm{~nm}$ laser pulse.

temperature. The wavelengths of the absorption peaks of AA, 3-EtAA, TX and NA are 265, 243, 289 and $262 \mathrm{~nm}$, respectively. The wavelength of the absorption peak of RF near $355 \mathrm{~nm}$ is $373 \mathrm{~nm}$. As is clearly seen in Fig. 1, only RF is excited through the $355 \mathrm{~nm}$ laser excitation in the mixtures of $0.1 \mathrm{mmol}$ $\mathrm{dm}^{-3} \mathrm{RF}$ and $0.4 \mathrm{mmol} \mathrm{dm}^{-3} \mathrm{AA}, 3$-EtAA, TX or NA.

\subsection{Near-IR phosphorescence of singlet oxygen}

Quenching of the excited triplet states of some organic compounds by ground-state oxygen, ${ }^{3} \mathrm{O}_{2}\left({ }^{3} \Sigma_{\mathrm{g}}{ }^{-}\right)$, results in the photosensitized generation of singlet oxygen [9]. On the other hand, quenching of singlet oxygen by ground states of some organic compounds shortens the singlet oxygen lifetime [9].

The time-resolved near-IR phosphorescence spectra of singlet oxygen by photosensitization with RF in the absence and presence of AA, 3-EtAA and Trolox were measured in air-saturated phosphate buffer at $25^{\circ} \mathrm{C}$. The results are shown in Fig. 2. The phosphorescence peak was observed at the same wavelength as it was observed in $\mathrm{H}_{2} \mathrm{O}$ by Wessels and Rodgers, $1274 \mathrm{~nm}$ [29].

Time profiles of the phosphorescence intensity of singlet oxygen generated by RF in the absence and presence of AA, 3-EtAA, Trolox and NA were measured, as shown in Fig. 3. To eliminate the background (probably residual contribution of delayed fluorescence from the photosensitizer) from the phosphorescence of singlet
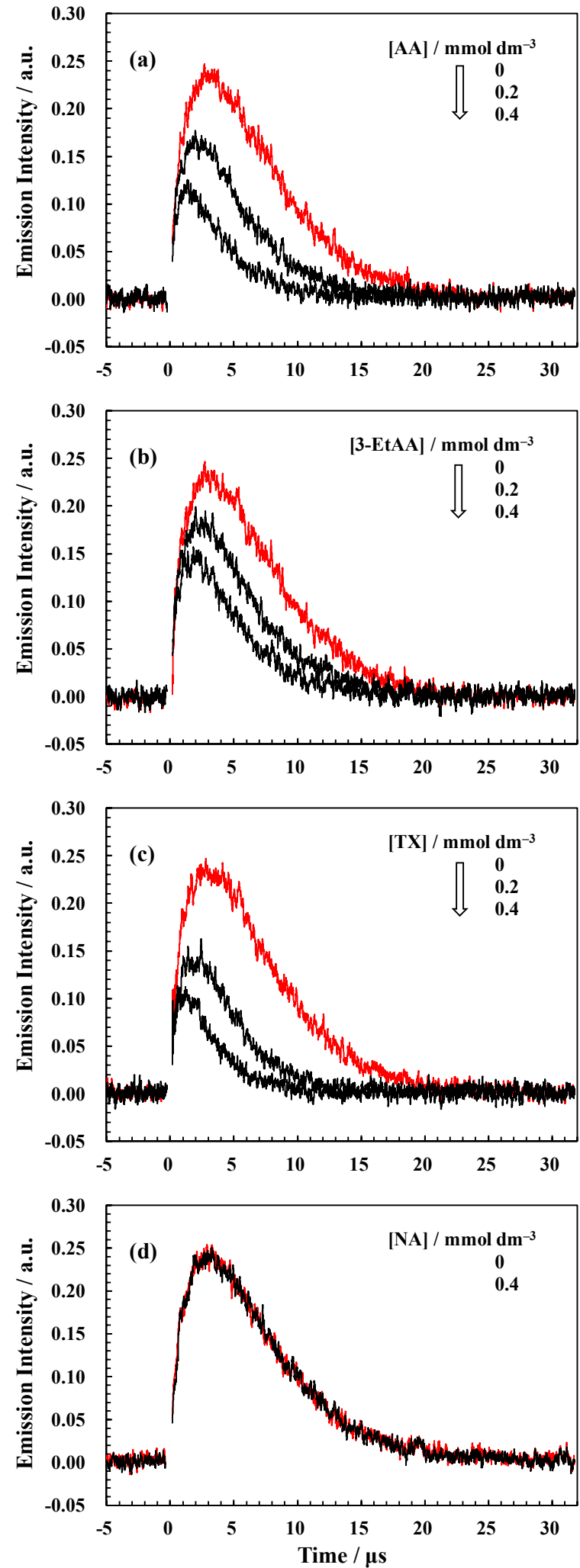

Fig. 3. Time profiles of the phosphorescence intensity of singlet oxygen taken following $355 \mathrm{~nm}$ laser excitation of RF $(0.1 \mathrm{mmol}$ $\left.\mathrm{dm}^{-3}\right)$ in air-saturated phosphate buffer $(\mathrm{pH} 6.8)$ at $25{ }^{\circ} \mathrm{C}$ in the absence (red) and presence of (a) AA, (b) 3-EtAA, (c) TX and (d) NA. The phosphorescence intensity was monitored at $1274 \mathrm{~nm}$.

oxygen, the average of the time profiles at $1200 \mathrm{~nm}$ and $1350 \mathrm{~nm}$ were subtracted from the time profile at $1274 \mathrm{~nm}$. 
While the lifetime of singlet oxygen slightly changes in the presence of AA, 3-EtAA and Trolox, the maximum phosphorescence intensity of singlet oxygen at $t_{\max }$ after the laser pulse remarkably decreases. As shown in Figs. $3 \mathrm{a}-3 \mathrm{c}, t_{\max }$ shortens in the presence of AA, 3-EtAA and Trolox. On the other hand, the time profiles of the phosphorescence intensity in the presence and presence of NA are exactly the same, as shown in Fig. $3 d$.

In a nanosecond pulsed laser excitation experiment, time profile of the detected phosphorescence signal, $I_{t}$, is described by the following equation:

$$
I_{t}=c \times \Phi_{\Delta} \times \tau_{\Delta} /\left(\tau_{\Delta}-\tau_{\mathrm{T}}\right) \times\left[\exp \left(-t / \tau_{\Delta}\right)-\exp \left(-t / \tau_{\mathrm{T}}\right)\right]
$$

where $c$ is a proportionality constant; $\Phi_{\Delta}$ is a quantum yield of singlet oxygen generation; $\tau_{\Delta}$ is a singlet oxygen lifetime; $\tau_{\mathrm{T}}$ is a lifetime of the triplet state of the photosensitizer in the presence of oxygen $\left(\tau_{\mathrm{T}} \neq \tau_{\Delta}\right)$ $[23,30,31]$. In Eq. (1), $I_{t}$ reaches a relative maximum at $t_{\text {max }}$ :

$$
t_{\max }=\left(\ln \tau_{\Delta}-\ln \tau_{\mathrm{T}}\right) /\left(\tau_{\mathrm{T}}^{-1}-\tau_{\Delta}^{-1}\right) .
$$

It should be noted that even if the value of $\Phi_{\Delta}$ is the same, the maximum phosphorescence intensity of singlet oxygen depends on $\tau_{\Delta}$ and $\tau_{\mathrm{T}}$. For example, when $\tau_{\Delta}$ is constant, the maximum intensity increases and $t_{\max }$ shortens with decreasing $\tau_{\mathrm{T}}$. On the other hand, when $\tau_{\mathrm{T}}$ is constant, the maximum intensity decreases and $t_{\max }$ shortens with decreasing $\tau_{\Delta}$. The problem we are faced with is the assignment of the observed decrement of the maximum phosphorescence intensity of singlet oxygen to the suppression of singlet oxygen and/or to the decrement of $\tau_{\Delta}$. In order to solve the problem, lifetimes of the triplet state of RF, $\tau_{\mathrm{T}}$, in the absence and presence of the quenchers were determined through the measurements of triplet-triplet absorption.

\subsection{Triplet-triplet absorption of $R F$}

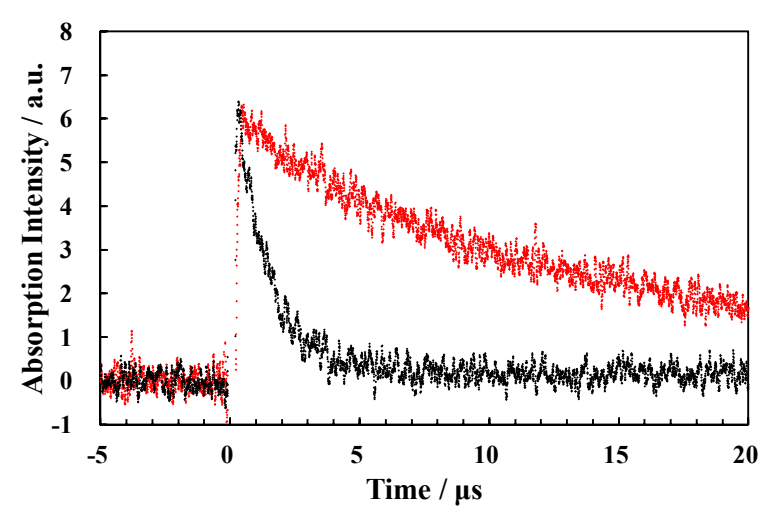

Fig. 4. (a) Time profiles of the transient absorption of RF $(0.1 \mathrm{mmol}$ $\mathrm{dm}^{-3}$ ) obtained following $355 \mathrm{~nm}$ laser excitation in $\mathrm{N}_{2}$-saturated phosphate buffer ( $\mathrm{pH} 6.8$ ) at $25{ }^{\circ} \mathrm{C}$ in the absence (red) and presence of $0.4 \mathrm{mmol} \mathrm{dm} \mathrm{dm}^{-3} \mathrm{TX}$. The transient absorption intensity was monitored at $690 \mathrm{~nm}$.

It is well known that the triplet-triplet absorption

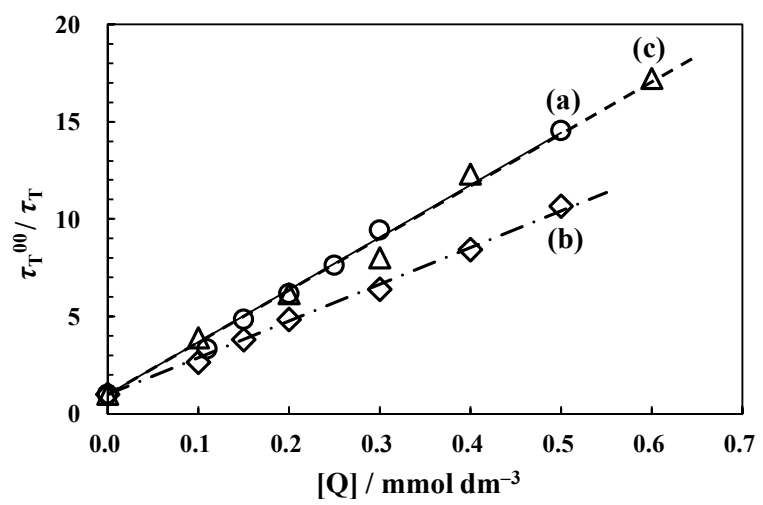

Fig. 5. Stern-Volmer plots according to Eq. (2) for the quenching of the triplet state of RF by (a) AA (solid line), (b) 3-EtAA (dash-dotdash line) and (c) TX (broken line) in nitrogen-saturated phosphate buffer (pH 6.8) at $25^{\circ} \mathrm{C}$.

spectrum of RF in aqueous solution has a broad absorption band between 500 and $800 \mathrm{~nm}$ [33,35-38]. Time profiles of the triplet-triplet absorption of RF obtained following $355 \mathrm{~nm}$ laser excitation in air-saturated phosphate buffer were measured in the absence and presence of AA, 3EtAA and TX, as shown in Figs. 4, S1 and S2.

The bimolecular rate constants for quenching of the triplet state of RF by the quenchers were estimated using the following Stern-Volmer equation [9]:

$$
\tau_{\mathrm{T}}^{00} / \tau_{\mathrm{T}}=1+\tau_{\mathrm{T}}{ }^{003} k_{\mathrm{q}}[\mathrm{Q}]
$$

where $\tau_{\mathrm{T}}{ }^{00}$ and $\tau_{\mathrm{T}}$ are the lifetimes of the triplet state of RF in the absence and presence of a quencher in nitrogen- 
saturated solutions, respectively; ${ }^{3} k_{\mathrm{q}}$ is the bimolecular rate constant for quenching of the triplet state of RF by a quencher; $[\mathrm{Q}]$ is the concentration of the quencher $\mathrm{Q}$.

The lifetimes of the triplet state of RF were measured as a function of the concentration of a quencher. The value of $\tau_{\mathrm{T}}{ }^{00}$ was obtained to be $16 \mu \mathrm{s}$ in nitrogen-saturated phosphate buffer. The values of $\tau_{\mathrm{T}}{ }^{00}$ divided by $\tau_{\mathrm{T}}$ were plotted against the quencher concentration, as shown in Fig. 5. The experimental values of ${ }^{3} k_{\mathrm{q}}$ were determined using the Stern-Volmer analysis of Eq. (3) and are listed in Table 1. The quenching of the triplet state of RF by AA and TX in aqueous buffer ( $\mathrm{pH}$ 6.4) was observed by Cardoso et al. [39]. The quenching of the excited singlet and triplet states of RF by TX in aqueous buffer ( $\mathrm{pH} 7)$ was observed by Gutiérrez et al. [40]. The quenching of the triplet state of RF by TX in aqueous solution was also observed by Yettella and Min [41]. The ${ }^{3} k_{\mathrm{q}}$ value of ground-state oxygen is listed for comparison. The lifetime of the triplet state of RF in the absence of a quencher in air-saturated phosphate buffer, $\tau_{\mathrm{T}}{ }^{0}$, was determined to be $3.0 \mu \mathrm{s}$.

As can be seen in Table 1, the quenching of the triplet

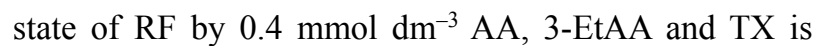

\section{Table 1}

Rate constants for quenching of excited singlet $\left({ }^{1} k_{\mathrm{q}}\right.$ in $\left.10^{9} \mathrm{~mol}^{-1} \mathrm{dm}^{3} \mathrm{~s}^{-1}\right)$ and triplet $\left({ }^{3} k_{\mathrm{q}}\right.$ in $\left.10^{9} \mathrm{~mol}^{-1} \mathrm{dm}^{3} \mathrm{~s}^{-1}\right)$ states of RF and for quenching of singlet oxygen $\left({ }^{\Delta} k_{\mathrm{q}}\right.$ in $\left.10^{8} \mathrm{~mol}^{-1} \mathrm{dm}^{3} \mathrm{~s}^{-1}\right)$ by quenchers. Quantum yields of RF-photosensitized singlet oxygen generation $\left(\Phi_{\Delta}\right)$ in the presence of $0.4 \mathrm{mmol} \mathrm{dm}^{-3}$ AA, 3-EtAA and TX.

\begin{tabular}{lccll}
\hline Quencher & ${ }^{1} k_{\mathrm{q}}$ & ${ }^{3} k_{\mathrm{q}}$ & ${ }^{\Delta} k_{\mathrm{q}}$ & $\Phi_{\Delta}{ }^{\mathrm{a}}$ \\
\hline AA & $4.0^{\mathrm{b}}$ & $1.6^{\mathrm{b}}$ & $1.8^{\mathrm{b}}$ & $0.20^{\mathrm{b}}$ \\
3-EtAA & $2.2^{\mathrm{b}}$ & $1.2^{\mathrm{b}}$ & $0.27^{\mathrm{c}}$ & $0.25^{\mathrm{b}}$ \\
TX & $5.4^{\mathrm{b}}$ & $1.6^{\mathrm{b}}$ & $4.4^{\mathrm{b}}$ & $0.20^{\mathrm{b}}$ \\
& & & & \\
Oxygen $\left({ }^{3} \mathrm{O}_{2}\right)$ & $4.0^{\mathrm{d}}$ & $1.2^{\mathrm{e}}$ & $0.000041^{\mathrm{f}}$ & - \\
\hline
\end{tabular}

a The value of $\Phi_{\Delta}$ in the absence of a suppressor was assumed to be 0.60 [1].

b This work, in phosphate buffer ( $\mathrm{pH}$ 6.8).

This work, in phosphate buffer ( $\mathrm{pH}$ 6.8)-acetonitrile (1:1 by volume).

${ }^{\mathrm{d}}$ From [32], in phosphate buffer ( $\left.\mathrm{pH} 7.0\right)$.

${ }^{\mathrm{e}}$ From [33], in phosphate buffer $(\mathrm{pH} 7)$

${ }^{\mathrm{f}}$ From [34], in perfluorodecalin.

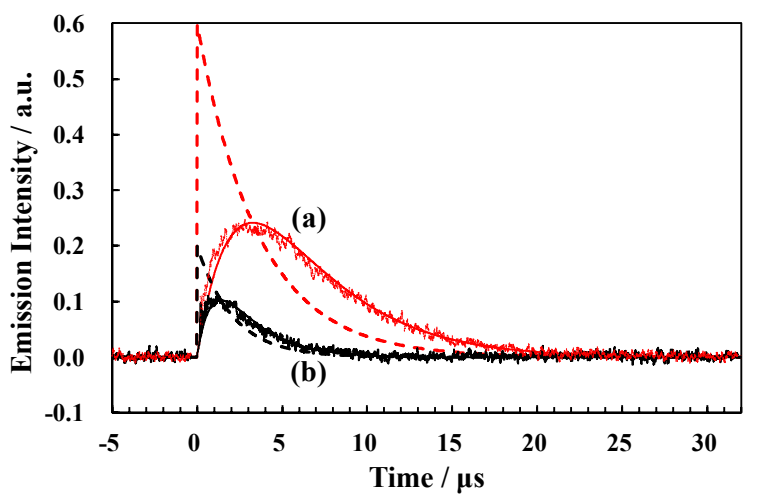

Fig. 6. Time profiles of the phosphorescence intensity of singlet oxygen generated by excitation of RF $\left(0.1 \mathrm{mmol} \mathrm{dm}^{-3}\right)$ (a) in the absence (red) and (b) presence of TX $\left(0.4 \mathrm{mmol} \mathrm{dm}^{-3}\right)$ in air-saturated phosphate buffer ( $\mathrm{pH} \mathrm{6.8)}$ at $25^{\circ} \mathrm{C}$. Computer-simulated time profiles obtained with (a) $c=1.0, \Phi_{\Delta}=0.60, \tau_{\Delta}=3.6 \mu \mathrm{s}, \tau_{\mathrm{T}}=0.001 \mu \mathrm{s}$ (broken line) and $\tau_{\mathrm{T}}=3.0 \mu \mathrm{s}$ (solid line), and (b) $c=1.0, \Phi_{\Delta}=0.20, \tau_{\Delta}=2.1$ $\mu \mathrm{s}, \tau_{\mathrm{T}}=0.001 \mu \mathrm{s}$ (broken line) and $\tau_{\mathrm{T}}=1.0 \mu \mathrm{s}$ (solid line).

competitive with that by ground-state oxygen in airsaturated aqueous solutions because the concentration of oxygen in water is $0.27 \mathrm{mmol} \mathrm{dm}{ }^{-3}$ at $25^{\circ} \mathrm{C}$ [42].

\subsection{Computer-simulated time profile of phosphorescence} intensity of singlet oxygen

The $\tau_{\mathrm{T}}$ values in the presence of $0.4 \mathrm{mmol} \mathrm{dm}^{-3} \mathrm{AA}, 3-$ EtAA and TX were determined to be 1.0, 1.2 and $1.0 \mu \mathrm{s}$, respectively. The observed time profiles of the phosphorescence intensity of singlet oxygen were simulated using the observed $\tau_{\mathrm{T}}$ values. The results are shown in Figs. 6, S3 and S4. As is seen in Fig. 6, the decrement of the maximum phosphorescence intensity of singlet oxygen in the presence of TX is not due to the decrement of $\tau_{\Delta}$. This is due to the decrement of $\Phi_{\Delta}$. For example, the $\Phi_{\Delta}$ value is decreased to a third by adding $0.4 \mathrm{mmol} \mathrm{dm}^{-3} \mathrm{TX}$ in an aqueous solution. The computersimulated $t_{\max }$ value is shortened from $3.3 \mu \mathrm{s}$ to $1.4 \mu \mathrm{s}$. These computer-simulated $t_{\max }$ values are close to the observed values as shown in Figs. $3 \mathrm{c}$ and 6 . The $\tau_{\Delta}$ value is shortened from $\tau_{\Delta}^{0}=3.6 \mu$ s to $\tau_{\Delta}=2.1 \mu \mathrm{s}$. In the same manner as for TX, we can see that the value of $\Phi_{\Delta}$ decreases in the presence of AA and 3-EtAA, as shown in Figs. S3 and S4. The values of $\Phi_{\Delta}$ in the presence of 0.4 mmol $\mathrm{dm}^{-3}$ AA, 3-EtAA and TX are listed in Table 1. 


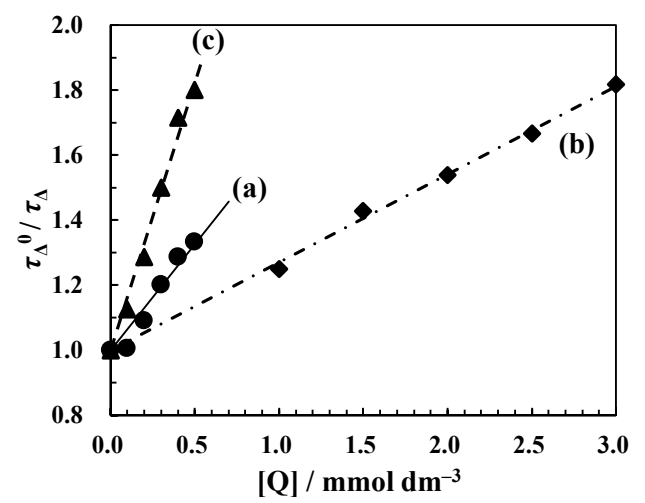

Fig. 7. Stern-Volmer plots according to Eq. (4) for the quenching of singlet oxygen by (a) AA and (c) TX in phosphate buffer ( $\mathrm{pH} 6.8$ ), and (b) 3-EtAA in phosphate buffer (pH 6.8)-acetonitrile (1:1 by volume) at $25^{\circ} \mathrm{C}$.

These facts show that AA, 3-EtAA and TX suppress the RF-photosensitized singlet oxygen generation in airsaturated aqueous solutions. As is seen in Table 1, AA and TX are more efficient suppressors of RF-photoinduced singlet oxygen generation than 3-EtAA.

Jockush et al. reported that protoporphyrin IXphotosensitized singlet oxygen generation is suppressed by the cyanoacrylates with fused aromatic rings in acetonitrile and chloroform [43]. It should be mentioned here that in this study the suppressors are vitamins and a vitamin derivative approved as safe for humans. The solvent used in this study is a buffer solution ( $\mathrm{pH} 6.8$ ).

\subsection{Quenching of singlet oxygen}

The rate constants for the quenching of singlet oxygen by AA, 3-EtAA and TX were estimated using the following Stern-Volmer equation [9]:

$$
\tau_{\Delta}{ }^{0} \tau_{\Delta}=1+\tau_{\Delta}{ }^{0}{ }^{\Delta} k_{\mathrm{q}}[\mathrm{Q}]
$$

where $\tau_{\Delta}{ }^{0}$ and $\tau_{\Delta}$ are the lifetimes of singlet oxygen in the absence and presence of a quencher respectively; ${ }^{\Delta} k_{\mathrm{q}}$ is the bimolecular rate constant for quenching of singlet oxygen by a quencher Q. As is seen in Eq. (1), it is difficult to estimate the value of $\tau_{\Delta}$ from the decay part of a time profile when the values of $\tau_{\Delta}$ and $\tau_{\mathrm{T}}$ are close. The $\tau_{\Delta}{ }^{0}$ value in air-saturated water is $3.6 \mu \mathrm{s}$ [23]. The $\tau_{\mathrm{T}}{ }^{0}$ value of RF in

$$
\begin{aligned}
& \mathrm{RF}+h v \longrightarrow{ }^{1} \mathrm{RF}^{*} \\
& { }^{1} \mathrm{RF}^{*} \underset{k_{\mathrm{F}}}{\mathrm{RF}}+h v^{\prime} \\
& { }^{1} \mathrm{RF}^{*} \underset{k_{\mathrm{IC}}}{\mathrm{RF}} \\
& { }^{1} \mathrm{RF}^{*} \underset{{ }^{1} k_{\mathrm{ISC}}}{\longrightarrow}{ }^{3} \mathrm{RF}^{*} \\
& { }^{1} \mathrm{RF}^{*}+{ }^{3} \mathrm{O}_{2} \underset{{ }^{1} k_{\mathrm{q}} \mathrm{O}_{2}}{\longrightarrow} \mathrm{RF}+{ }^{3} \mathrm{O}_{2} \\
& { }^{1} \mathrm{RF}^{*}+\mathrm{Q} \underset{{ }^{1} k_{\mathrm{q}} \mathrm{Q}}{\longrightarrow} \mathrm{RF}+\mathrm{Q} \\
& { }^{3} \mathrm{RF}^{*} \underset{{ }^{3} k_{\mathrm{ISC}}}{\longrightarrow} \mathrm{RF} \\
& { }^{3} \mathrm{RF}^{*}+{ }^{3} \mathrm{O}_{2} \underset{{ }^{3} \mathrm{q} \mathrm{O}_{2}}{\longrightarrow} \mathrm{RF}+{ }^{1} \mathrm{O}_{2} \\
& { }^{3} \mathrm{RF}^{*}+\mathrm{Q} \underset{{ }^{3} k_{\mathrm{q}} \mathrm{Q}}{\mathrm{RF}}+\mathrm{Q} \\
& { }^{1} \mathrm{O}_{2} \stackrel{ }{{ }^{4} k_{\mathrm{d}}}{ }^{3} \mathrm{O}_{2} \\
& { }^{1} \mathrm{O}_{2}+\mathrm{Q} \underset{{ }_{k_{\mathrm{q}}}{ }^{\mathrm{s}}}{\longrightarrow}{ }^{3} \mathrm{O}_{2}+\mathrm{Q}
\end{aligned}
$$

Scheme 2. kinetics of the excited singlet and triplet states of RF and singlet oxygen.

air-saturated water was determined to be are $3.0 \mu \mathrm{s}$ through the measurements of triplet-triplet absorption mentioned in section 3.3 .

The $\tau_{\Delta}$ values were estimated from the computersimulated time profiles of phosphorescence intensity of singlet oxygen mentioned in section 3.4. The values of $\tau_{\Delta}{ }^{0}$ divided by $\tau_{\Delta}$ were plotted against the quencher concentration, as shown in Fig. 7. For 3-EtAA, the time profiles were measured in phosphate buffer ( $\mathrm{pH}$ 6.8)acetonitrile (1:1 by volume) because ${ }^{\Delta} k_{\mathrm{q}}$ by 3 -EtAA is small and $\tau_{\Delta}$ in the phosphate buffer-acetonitrile mixture is longer than that in phosphate buffer. The results are shown in Fig. S5. The $\tau_{\Delta}{ }^{0}$ value in the phosphate bufferacetonitrile mixture was determined to be $10 \mu \mathrm{s}$. As is shown in Fig. $7,{ }^{\Delta} k_{\mathrm{q}}$ decreases in the following order: ${ }^{\Delta} k_{\mathrm{q}}{ }^{\mathrm{TX}}$ ${ }^{\Delta} k_{\mathrm{q}}{ }^{\mathrm{AA}}>{ }^{\Delta} k_{\mathrm{q}}{ }^{3 \text {-EtAA }}$. The ${ }^{\Delta} k_{\mathrm{q}}$ values were determined using the Stern-Volmer analysis of Eq. (4) and are listed in Table 1 . The ${ }^{\Delta} k_{\mathrm{q}}$ value by ground state oxygen listed in Table 1 




Fig. 8. Stern-Volmer plots according to Eq. (5) for the quenching of the excited singlet state of RF by (a) AA (solid line), (b) 3-EtAA (dash-dot-dash line) and (c) TX (broken line) in air-saturated phosphate buffer ( $\mathrm{pH} 6.8)$ at $25^{\circ} \mathrm{C}$.

was determined in perfluorodecalin because ${ }^{\Delta} k_{\mathrm{q}}$ is small and $\tau_{\Delta}$ in air-saturated perfluorodecalin is long, $60 \mathrm{~ms}$ [34].

\subsection{Quenching of fluorescence of $R F$}

Possible mechanisms of the suppression of RFphotosensitized singlet oxygen generation involve quenching of the excited singlet and triplet states of RF. As is shown in Scheme 2, in the presence of oxygen and a quencher, there are additionally competitive kinetics of the quenching of the excited singlet and triplet states of RF by ground-state oxygen and a quencher. The quenching of the excited singlet state by a quencher competes with ${ }^{1} \mathrm{RF}^{*} \rightarrow{ }^{3} \mathrm{RF}^{*}$ intersystem crossing, ${ }^{1} k_{\mathrm{ISC}}$ in Scheme 2, and decreases a quantum yield of the triplet state generation in air-saturated solutions, resulting in the decrement of $\Phi_{\Delta}$. The quenching of the triplet state by a quencher also decreases $\Phi_{\Delta}$ in air-saturated solutions.

The bimolecular quenching rate constants for the excited singlet state of RF by AA, 3-EtAA and TX were determined by measuring the fluorescence lifetimes of RF. The bimolecular rate constants were estimated using the following Stern-Volmer equations:

$$
\tau_{\mathrm{F}}^{0} / \tau_{\mathrm{F}}=1+\tau_{\mathrm{F}}{ }^{0} k_{\mathrm{q}}[\mathrm{Q}]
$$

where $\tau_{\mathrm{F}}{ }^{0}$ and $\tau_{\mathrm{F}}$ are the fluorescence lifetimes of RF in the absence and presence of a quencher in air-saturated solutions, respectively; ${ }^{1} k_{\mathrm{q}}$ is the bimolecular rate constant for quenching of the excited singlet state of RF by the quencher Q [9].

Stern-Volmer analysis shown in Fig. 8 was performed to quantify the quenching of fluorescence by the quenchers. The observed $\tau_{\mathrm{F}}{ }^{0}$ value is $4.80 \mathrm{~ns}$ in phosphate buffer ( $\mathrm{pH}$ 6.8). The $\tau_{\mathrm{F}}^{0}$ value observed in the present study is in good agreement with the previously reported values, $4.7 \mathrm{~ns}$ (in $\mathrm{pH} 7.6$ phosphate buffer) [44] and 5.06 ns (in pH 7.0 aqueous solution) [45]. From the slope of the Stern-Volmer plots the bimolecular rate constants for quenching of the excited singlet state of RF by the quenchers $\left({ }^{1} k_{\mathrm{q}}\right)$ were determined and listed in Table 1.

\subsection{Effects of quenching of the excited states of RF on} singlet oxygen generation

As can be seen in Table 1, the quenching rate constants of the excited singlet state of RF by AA, 3-EtAA and TX are comparable to that by ground-state oxygen. Generation of singlet oxygen from the excited singlet state of a sensitizer is possible providing that the singlet-triplet energy gap exceeds the energy required to excite groundstate oxygen, $7800 \mathrm{~cm}^{-1}$, and singlet lifetime is long enough for oxygen to quench it $[9,23]$. The singlet-triplet energy gap of RF, $2700 \mathrm{~cm}^{-1}$, is smaller than the energy to excite ground state oxygen $[42,46]$. In most cases, singlet oxygen generates exclusively from the triplet state of the sensitizer $[9,23]$.

Several rate constants in Scheme 2 and quantum yields were estimated using the following equations:

$$
\begin{aligned}
& { }^{1} k_{\mathrm{ISC}}=\Phi_{\mathrm{T}}{ }^{00} / \tau_{\mathrm{F}}{ }^{00} \\
& { }^{3} k_{\mathrm{ISC}}=1 / \tau_{\mathrm{T}}^{00} \\
& \Phi_{\mathrm{T}}={ }^{1} k_{\mathrm{ISC}} /\left(k_{\mathrm{F}}+k_{\mathrm{IC}}+{ }^{1} k_{\mathrm{ISC}}+{ }^{1} k_{\mathrm{q}} \mathrm{O}_{2}\left[\mathrm{O}_{2}\right]+{ }^{1} k_{\mathrm{q}}^{\mathrm{Q}}[\mathrm{Q}]\right) \\
& ={ }^{1} k_{\mathrm{ISC}} /\left(1 / \tau_{\mathrm{F}}{ }^{00}+{ }^{1} k_{\mathrm{q}}{ }^{\mathrm{O} 2}\left[\mathrm{O}_{2}\right]+{ }^{1} k_{\mathrm{q}}{ }^{\mathrm{Q}}[\mathrm{Q}]\right)
\end{aligned}
$$

$$
\Phi_{\Delta}=\Phi_{\mathrm{T}}{ }^{3} k_{\mathrm{q}} \mathrm{O}_{2}\left[\mathrm{O}_{2}\right] /\left({ }^{3} k_{\mathrm{ISC}}+{ }^{3} k_{\mathrm{q}} \mathrm{O}_{2}\left[\mathrm{O}_{2}\right]+{ }^{3} k_{\mathrm{q}} \mathrm{Q}[\mathrm{Q}]\right)
$$


where $\Phi_{\mathrm{T}}{ }^{00}$ and $\Phi_{\mathrm{T}}$ are the quantum yields of triplet generation in the absence and presence of a quencher and oxygen, respectively; $\tau_{\mathrm{F}}{ }^{00}$ is the fluorescence lifetime in the absence of a quencher and oxygen. In RFphotosensitized singlet oxygen generation, the quantum yield of triplet generation $\left(\Phi_{\mathrm{T}}^{0} \approx 0.6\right)$ is similar to that of singlet oxygen generation $\left(\Phi_{\Delta}=0.58-0.61\right)[1,36,47-49]$. We can safely assume that the fraction of triplet quenching by ground-state oxygen that results in the formation of singlet oxygen is unity in Eq. (9) [9].

The fluorescence lifetime of RF in Ar-saturated phosphate buffer ( $\mathrm{pH} 6.8), \tau_{\mathrm{F}}^{00}=4.95 \mathrm{~ns}$, is close to that in air-saturated phosphate buffer, $\tau_{\mathrm{F}}{ }^{0}=4.80 \mathrm{~ns}$. We can safely assume that the effect of ground-state oxygen on $\Phi_{\mathrm{T}}$ is small. The rate constant of ${ }^{1} k_{\mathrm{ISC}}$ of ${ }^{1} \mathrm{RF}^{*}$ was estimated to $\mathrm{be} \approx 1.2 \times 10^{8} \mathrm{~s}^{-1}$ from the fluorescence lifetime of RF in Ar-saturated phosphate buffer and quantum yield of the triplet state generation of RF, $\Phi_{\mathrm{T}}^{00} \approx \Phi_{\mathrm{T}}^{0} \approx 0.6[36,47-49]$. The quenching rates of the excited singlet state of RF by ground-state oxygen and $0.4 \mathrm{mmol} \mathrm{\textrm {dm } ^ { - 3 }}$ TX was estimated to be $1.1 \times 10^{6} \mathrm{~s}^{-1}$ and $2.2 \times 10^{6} \mathrm{~s}^{-1}$, respectively. The estimated $\Phi_{\mathrm{T}}$ value using Eq. (8) in air-saturated phosphate aqueous solutions in the presence of $0.4 \mathrm{mmol}$ $\mathrm{dm}^{-3} \mathrm{TX}, 0.60$, is the same as that in the absence of TX, $\approx$ 0.6. The effects of the quenching of the excited singlet state of RF by TX on $\Phi_{\mathrm{T}}$ and $\Phi_{\Delta}$ are small in air-saturated water when the concentration of TX is close to that of dissolved oxygen in water. The concentration of TX required to reduce $\Phi_{\mathrm{T}}$ by half, $\Phi_{\mathrm{T}}=0.30$, was estimated to be $38 \mathrm{mmol} \mathrm{dm}^{-3}$ using Eq. (8).

The rate constant of ${ }^{3} \mathrm{RF}^{*} \rightarrow \mathrm{RF}$ intersystem crossing, ${ }^{3} k_{\mathrm{ISC}}$ in Scheme 2, was estimated to be $8 \times 10^{3} \mathrm{~s}^{-1}$ from the triplet lifetime of RF in oxygen-free water, $\tau_{\mathrm{T}}{ }^{00}=120 \mu \mathrm{s}$ [48]. The quenching rate of the triplet state of RF by 0.4 mmol dm ${ }^{-3}$ TX was estimated to be $6.4 \times 10^{5} \mathrm{~s}^{-1}$. The quenching rate of the triplet state of RF by ground-state oxygen was estimated to be $3.2 \times 10^{5} \mathrm{~s}^{-1}$ from the concentration of oxygen in water. Using Eq. (9) the values of $\Phi_{\Delta}$ in the absence and presence of TX $\left(0.4 \mathrm{mmol} \mathrm{dm}^{-3}\right)$

were estimated to be 0.60 and 0.20 , respectively. The estimated value of 0.20 in the presence of TX $(0.4 \mathrm{mmol}$ $\mathrm{dm}^{-3}$ ) is in good agreement with the computer-simulated value of 0.20 shown in Fig. 6. Therefore, the suppression of RF-photosensitized singlet oxygen generation by TX is ascribed mainly to the quenching of the triplet state of RF when the concentration of TX is close to that of dissolved oxygen in water. The quenching of the excited singlet state of RF by TX also concerns the observed suppression of singlet oxygen generation to some degree. This situation is the same as for AA and 3-EtAA.

Although the observed rate constants for quenching of the excited singlet state of RF by AA, 3-EtAA and TX are larger than those for quenching of the triplet state, quenching of the excited singlet state makes a minor contribution to the suppression of singlet oxygen generation. This is due to the short lifetime of the excited singlet state.

The energy levels of the lowest excited singlet and triplet states of RF are lower than those of AA, 3-EtAA and TX [46]. The energy transfer from RF to AA, 3-EtAA and TX is energetically unlikely. The electron transfer is probably involved in the quenching mechanism. However, in laser excitation experiments no major photodecomposition was observed in the presence of AA, 3-EtAA and TX. The absorption spectra and phosphorescence intensities of singlet oxygen did not change in laser excitation experiments under our experimental conditions mentioned in the experimental section. One possible explanation for the absence of photodecomposition is that the electron transfer is followed by fast back-electron transfer recovering RF in the ground state. These situations are similar to the suppression of protoporphyrin IX-photosensitized singlet oxygen generation by the cyanoacrylates reported by Jockusch et al. [43].

\section{Conclusion}


The suppression of RF-photosensitized singlet oxygen generation by AA, 3-EtAA and TX was observed in air-saturated phosphate buffer $(\mathrm{pH}$ 6.8) at room temperature. The suppression can be ascribed mainly to the quenching of the triplet states of RF by AA, 3-EtAA and TX. The quenching of the excited singlet state of RF by AA, 3-EtAA and TX also concerns the observed suppression of singlet oxygen generation to some degree.

\section{Acknowledgements}

This work was supported in part by JSPS KAKENHI

Grant Number 24655060.

\section{References}

[1] W. Bäumler, Endogenous singlet oxygen photosensitizers in mammalians, in: S. Nonell, C. Flors (Eds.), Singlet Oxygen: Application in Biosciences and Nanosciences, The Royal Society of Chemistry, Cambridge, UK, 2016, vol. 1, pp. 225-238.

[2] D.R. Cardoso, S.H. Libardi, L.H. Skibsted, Riboflavin as a photosensitizer. Effects on human health and food quality, Food Funct. 3 (2012) 487-502.

[3] R. Haywood, C. Andrady, N. Kassouf, N. Sheppard, Intensity-dependent direct solar radiation- and UVAinduced radical damage to human skin and DNA, lipids and proteins, Photochem. Photobiol. 87 (2011) 117-130.

[4] G.T. Wondrak, M.K. Jacobson, E.L. Jacobson, Endogenous UVA-photosensitizers: mediators of skin photodamage and novel targets for skin photoprotection Photochem. Photobiol. Sci. 5 (2006) 215-237.

[5] G. Viteri, A.M. Edwards, J. De la Fuente, E. Silva, Study of the interaction between triplet riboflavin and the $\alpha$-,

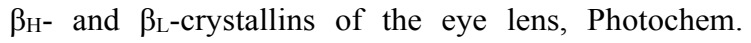
Photobiol. 77 (2003) 535-540.

[6] W. Bäumler, Singlet oxygen in the skin, in: S. Nonell, C. Flors (Eds.), Singlet Oxygen: Application in Biosciences and Nanosciences, The Royal Society of Chemistry, Cambridge, UK, 2016, vol. 2, pp. 205-226.

[7] J. Roberts, B. Zhao, Singlet oxygen in the eye, in: S. Nonell, C. Flors (Eds.), Singlet Oxygen: Application in Biosciences and Nanosciences, The Royal Society of Chemistry, Cambridge, UK, 2016, vol. 2, pp. 227-249.

[8] E. Boix-Garriga, B. Rodríguez-Amigo, O. Planas, S. Nonell, Properties of singlet oxygen, in: S. Nonell, C. Flors (Eds.), Singlet Oxygen: Application in Biosciences and Nanosciences, The Royal Society of Chemistry, Cambridge, UK, 2016, vol. 1, pp. 23-46.
[9] N.J. Turro, V. Ramamurthy, J.C. Scaiano, Modern Molecular Photochemistry of Organic Molecules, University Science Books, Sausalito, USA, 2010.

[10] C. Schweitzer, R. Schmidt, Physical mechanisms of generation and deactivation of singlet oxygen, Chem. Rev. 103 (2003) 1685-1757.

[11] F. Wilkinson, W.P. Helman, A.B. Ross, Rate constants for the decay and reactions of the lowest electronically excited singlet state of molecular oxygen in solution. An expanded and revised compilation, J. Phys. Chem. Ref. Data 24 (1995) 663-1021.

[12] J.M. King, D.B. Min, Riboflavin photosensitized singlet oxygen oxidation of vitamin D, J. Food Sci. 63 (1998) 31-34.

[13] M.Y. Jung, S.K. Kim, S.Y. Kim, Riboflavin-sensitized photooxidation of ascorbic acid: kinetics and amino acid effects, Food Chem. 53 (1995) 397-403.

[14] P.-T. Chou, A.U. Khan, L-ascorbic acid quenching of singlet delta molecular oxygen in aqueous media: Generalized antioxidant property of vitamin C, Biochem. Biophys. Res. Commun. 115 (1983) 932-937.

[15] K. Mukai, A. Ouchi, S. Takahashi, K. Aizawa, T. Inakuma, J. Terao, S. Nagaoka, Development of singlet oxygen absorption capacity (SOAC) assay method. 3 . Measurements of the SOAC values for phenolic antioxidants, J. Agric. Food Chem. 60 (2012) 79057916.

[16] J.B. Arellano, H. Li, S. González-Pérez, J. Gutiérrez, T.B Melø, F. Vacha, K.R. Naqvi, Trolox, a water-soluble analogue of $\alpha$-tocopherol, photoprotects the surfaceexposed regions of the photosystem II reaction center in vitro. Is this physiologically relevant?, Biochem. 50 (2011) 8291-8301.

[17] K. Ohara, K. Kikuchi, T. Origuchi, S. Nagaoka, Singlet oxygen quenching by trolox $\mathrm{C}$ in aqueous micelle solutions, J. Photochem. Photobiol. B 97 (2009) 132137.

[18] R.H. Bisby, C.G. Morgan, I. Hamblett, A.A. Gorman Quenching of singlet oxygen by Trolox C, ascorbate, and amino acids: effects of $\mathrm{pH}$ and temperature, J. Phys. Chem. A 103 (1999) 7454-7459.

[19] A. Yagami, K. Suzuki, Y. Morita, Y. Iwata, A. Sano, K. Matsunaga, Allergic contact dermatitis caused by 3-oethyl-L-ascorbic acid (vitamin C ethyl), Contact Dermatitis 70 (2014) 376-377.

[20] J. Hsu, 3-O-ethyl ascorbic acid: a stable vitamin Cderived agent for skin whitening, Cosmet. Toiletries 128 (2013) 676-684.

[21] A.M. Victoria-Martínez, P. Mercader-García, Allergic contact dermatitis to 3-o-ethyl-L-ascorbic acid in skinlightening, Dermatitis 28 (2017) 89.

[22] T. Numata, Y. Kobayashi, T. Ito, K. Harada, R. Tsuboi, Y. Okubo, Two cases of allergic contact dermatitis due to skin-whitening cosmetics, Allergol. Int. 64 (2015) 194-195.

[23] S. Nonell, C. Flors, Steady-state and time-resolved singlet oxygen phosphorescence detection in the near-IR, in: S. Nonell, C. Flors (Eds.), Singlet Oxygen: Applications in Biosciences and Nanosciences, The 
Royal Society of Chemistry, Cambridge, UK, 2016, vol. 2, pp. 7-26.

[24] R. Shimizu, M. Yagi, N. Oguchi-Fujiyama, K. Miyazawa, A. Kikuchi, Photophysical properties of diethylhexyl 2,6-naphthalate (Corapan TQ), a photostabilizer for sunscreens, Photochem. Photobiol. Sci. 17, (2018) 1206-1212.

[25] S. Matsumoto, R. Kumasaka, M. Yagi, A. Kikuchi, Triplet-triplet energy transfer between UV absorbers in solutions at room temperature, J. Photochem. Photobiol. A 346 (2017) 396-400.

[26] Y. Shamoto, M. Yagi, N. Oguchi-Fujiyama, K. Miyazawa, A. Kikuchi, Photophysical properties of hexyl diethylaminohydroxybenzoylbenzoate (Uvinul A Plus), a UV-A absorber, Photochem. Photobiol. Sci. 16 (2017) 1449-1457.

[27] A. Kikuchi, K. Shibata, R. Kumasaka, M. Yagi, Optical and time-resolved electron paramagnetic resonance studies of the excited states of a UV-B absorber (4methylbenzylidene)camphor, J. Phys. Chem. A 117 (2013) 1413-1419.

[28] A. Kikuchi, K. Shibata, R. Kumasaka, M. Yagi, Excited states of menthyl anthranilate: a UV-A absorber, Photochem. Photobiol. Sci. 12 (2013) 246-253.

[29] J.M. Wessels, M.A.J. Rodgers, Effect of solvent polarizability on the forbidden ${ }^{1} \Delta_{\mathrm{g}} \rightarrow{ }^{3} \Sigma_{\mathrm{g}}{ }^{-}$transition in molecular oxygen: a Fourier transform near-infrared luminescence study, J. Phys. Chem. 99 (1995) 1758617592.

[30] A.A. Krasnovsky Jr., Luminescence and photochemical studies of singlet oxygen photonics, J. Photochem. Photobiol. A 196 (2008) 210-218.

[31] J. Baier, T. Fuß, C. Pöllmann, C. Wiesmann, K. Pindl, R. Engl, D. Baumer, M. Maier, M. Landthaler, W. Bäumler, Theoretical and experimental analysis of the luminescence signal of singlet oxygen for different photosensitizers, J. Photochem. Photobiol. B 87 (2007) 163-173.

[32] J.R. Lakowicz, G. Weber, Quenching of fluorescence by oxygen. A probe for structural fluctuations in macromolecules, Biochemistry 12 (1973) 4161-4170.

[33] L. Valle, F.E. Morán Vieyra, C.D. Borsarelli, Nanoenvironmental effect in AOT reverse micelles on the triplet excited state properties of flavins and quenching by molecular oxygen, J. Phys. Org. Chem. 29 (2016) 629-635.

[34] E. Afshari, R. Schmidt, Isotope-dependent quenching of singlet molecular oxygen $\left({ }^{1} \Delta_{\mathrm{g}}\right)$ by ground-state oxygen in several perhalogenated solvents, Chem. Phys. Lett. 184 (1991) 128-132.

[35] L. de O.R. Arrivetti, R.S. Scurachio, W.G. Santos, T.B. R. Papa, L.H. Skibsted, D.R. Cardoso, Photooxidation of other B-vitamins as sensitized by riboflavin, J. Agric. Food Chem.61 (2013) 7615-7620.

[36] L. Valle, F.E. Morán Vieyra, C.D. Borsarelli, Hydrogenbonding modulation of excited-state properties of flavins in a model of aqueous confined environment, Photochem. Photobiol. Sci. 11 (2012) 1051-1061.
[37] S. Salzmann, V. Martinez-Junza, B. Zorn, S.E. Braslavsky, M. Mansurova, C. M. Marian, W. Gärtner, Photophysical properties of structurally and electronically modified flavin derivatives determined by spectroscopy and theoretical calculations, J. Phys. Chem. A 113 (2009) 9365-9375.

[38] K. Huvaere, K. Olsen, L.H. Skibsted, Quenching of triplet-excited flavins by flavonoids. Structural assessment of antioxidative activity, J. Org. Chem. 74 (2009) 7283-7293.

[39] D.R. Cardoso, K. Olsen, L.H. Skibsted, Mechanism of deactivation of triplet-excited riboflavin by ascorbate, carotenoids, and tocopherols in homogeneous and heterogeneous aqueous food model systems, J. Agric. Food Chem. 55 (2007) 6285-6291.

[40] I. Gutiérrez, S. Criado, S. Bertolotti, N.A. García, Dark and photoinduced interactions between Trolox, a polarsolvent-soluble model for vitamin E, and riboflavin, J. Photochem. Photobiol. B 62 (2001) 133-139.

[41] R.R. Yettella, D.B. Min, Quenching mechanisms and kinetics of Trolox and ascorbic acid on the riboflavinphotosensitized oxidation of tryptophan and tyrosine, J. Agric. Food Chem. 56 (2008) 10887-10892.

[42] M. Montalti, A. Credi, L. Prodi, M.T. Gandolfi, Handbook of Photochemistry, third ed., Taylor \& Francis, Boca Raton, 2006.

[43] S. Jockusch, C. Bonda, S. Hu, Photostabilization of endogenous porphyrins: excited state quenching by fused ring cyanoacrylates, Photochem. Photobiol. Sci., 13 (2014) 1180-1184.

[44] K. Hirakawa, T. Yoshioka, Photoexcited riboflavin induces oxidative damage to human serum albumin, Chem. Phys. Lett. 634 (2015) 221-224.

[45] P. Drössler, W. Holzer, A. Penzkofer, P. Hegemann, pH dependence of the absorption and emission behaviour of riboflavin in aqueous solution, Chem. Phys. 282 (2002) 429-439.

[46] R.W. Chambers, D.R. Kearns, Triplet states of some common photosensitizing dyes, Phtochem. Photobiol. 10 (1969) 215-219.

[47] L. Crovetto, V. Martínez-Junza, S.E. Braslavsky, Entropy changes drive the electron transfer reaction of triplet flavin mononucleotide from aromatic amino acids in cation-organized aqueous media. A Laser-induced optoacoustic study, Photochem. Photobiol. 82 (2006) 281-290.

[48] J. Baier, T. Maisch, M. Maier, E. Engel, M. Landthaler, W. Bäumler, Singlet oxygen generation by UVA light exposure of endogenous photosensitizers, Biophys. J. 91 (2006) 1452-1459.

[49] J.N. Chacon, J. McLearie, R.S. Sinclair, Singlet oxygen yields and radical contributions in the dye-sensitized photo-oxidation in methanol of esters of polyunsaturated fatty acids (oleic, linoleic and arachidonic), Photochem. Photobiol. 47 (1988) 647-656. 